\title{
Efficient Navigation Aware Seabed Coverage using AUVs
}

\author{
Özer Özkahraman and Petter Ögren
}

\begin{abstract}
Area coverage and robot navigation are two important research fields within robotics. However, their intersection has received limited attention. In coverage problems, perfect navigation is often assumed, and in robot navigation, the focus is often to minimize the localization error while traveling a given trajectory. The need for integration of the two becomes clear in environments with very sparse features or landmarks, for example when an Autonomous Underwater Vehicle (AUV) is to search the seafloor for dangerous objects, such as mines. The potential consequences of missing a mine due to navigation errors can be catastrophic. If the localization error is large, a trajectory that was designed to guarantee complete coverage might have missed significant parts of the area. Thus, the coverage trajectory must be planned with the navigation performance in mind, applying a combination of using large enough planned overlaps of sensor footprints to account for the position uncertainty, and reducing this uncertainty by revisiting the known sparse landmarks.

In this paper we compute trajectories that guarantee coverage for a given area under assumptions on worst case localization error growth. We further more compute upper bounds for how large areas can be covered using common coverage patterns and a single landmark, which leads to bounds on how sparse the landmarks can be in the regions to be covered.
\end{abstract}

\section{INTRODUCTION}

In this paper, we propose an approach for coverage in extremely landmark-sparse environments, using vehicles with significant navigation errors. Imagine a group of Autonomous Underwater Vehicles (AUVs) that are tasked to search the seafloor for objects such as mines. The sensor range is about $20 \mathrm{~m}$ but navigation is difficult due to the highly uniform seafloor, like an underwater sand desert. There are very sparse landmarks, such as larger rocks, but beyond those, the limited sensor range makes it impossible to tell the difference between two separate locations. The navigation system is therefore relying on compass and inertial measurement units (IMUs), resulting in an inevitable drift of slowly increasing navigation errors.

How can coverage be guaranteed, when the sensor range is $20 \mathrm{~m}$ and the absolute navigation error is $50 \mathrm{~m}$ ? The key observation is that the navigation error is both an absolute error, relative to the true vehicle position, and also a relative navigation error between any two vehicle positions. Thus, by carefully adapting the coverage pattern to a model of the error growth, and the location of the sparse existing landmarks, a partial coverage of the seabed can be guaranteed. Finally, for a given error growth, we can compute bounds on how sparse the landmarks can be, while still enabling complete coverage guarantees.

The problem is illustrated in Figure 1. The task is to cover a 300 by 300 meter area with no landmarks using an AUV

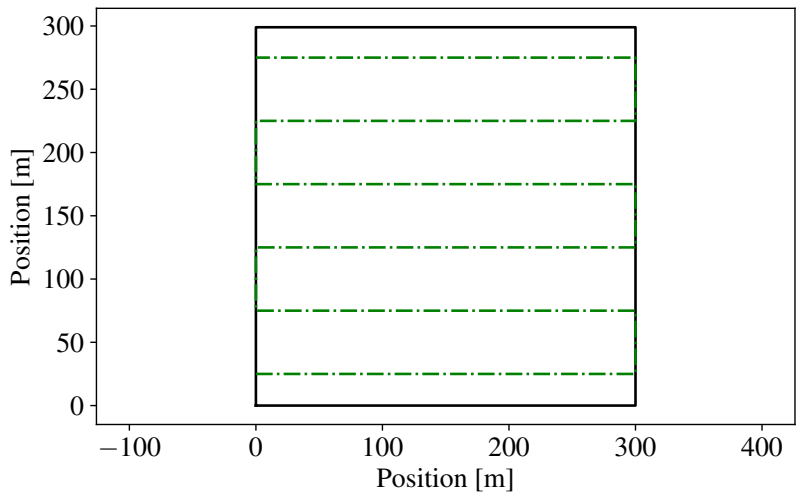

(a) Classical lawn mower pattern.

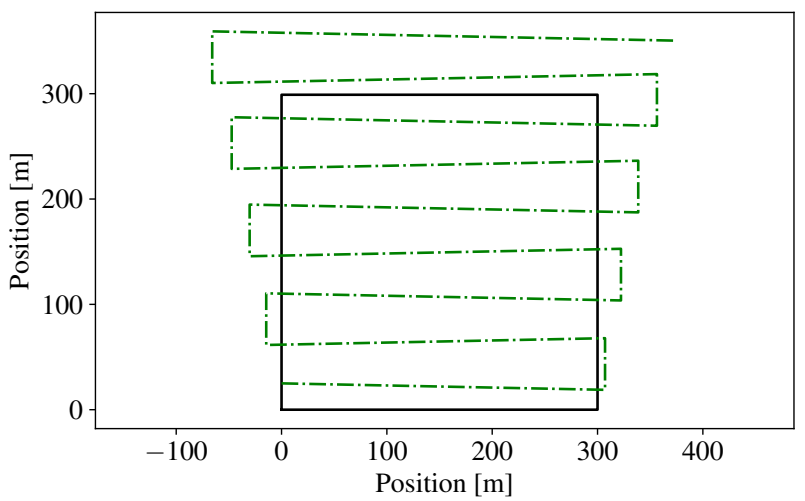

(b) Pattern adapted to small error growth.

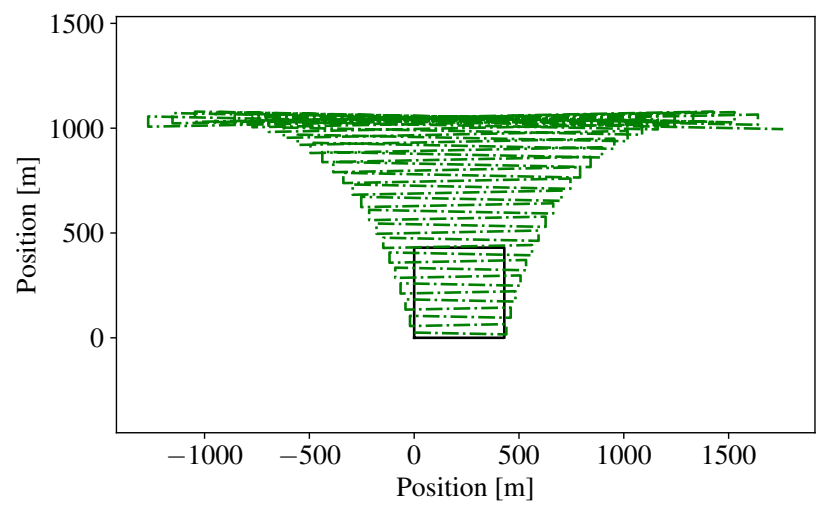

(c) For a larger square, adaption is impossible.

Fig. 1: The lawn mower coverage path plan and how it can be adapted to a growing navigation error. As can be seen in (c), there is a limit to how much navigation error can be handled. 
with a down-looking multi-beam sonar having a $20 \mathrm{~m}$ wide sensor footprint. Without navigation errors the solution is a classical lawn mower pattern, with $20 \mathrm{~m}$ sweep separation as can be seen in Figure 1(a). Assuming that an upper bound on the navigation error is given as a function of distance travelled, we can instead plan a trajectory similar to the one depicted in Figure 1(b). Note how each sweep must be longer than the previous, to take possible drift to the west and east into account. Furthermore, the sweep separation must be decreased to avoid gaps between two consecutive sweeps due to possible drift to the north, and additional sweeps added to counteract a drift to the south. As can be seen in Figure 1(c) there comes a point where the increase in sweep lengths leads to sweep separations tending to zero. This is due to the fact that these effects amplify each other where increasing errors lead to longer sweeps, which leads to smaller separation, which leads to increased number of sweeps needed to cover an area, leading to longer paths and increased errors and so on. In this paper we will explore this phenomenon, and provide quantitative results for how large areas can be covered and how efficient the coverage will be, given error growth, search patterns and landmark sparsity.

The main contribution of this paper is that we integrate the navigation error into the coverage path plan such that even when the navigation system is performing at its worse, complete coverage can still be guaranteed.

\section{RELATED WORK}

In [1], a coverage algorithm for autonomous surface vehicles is examined. This method takes into account the kinematics, modeled as Dubins vehicles, of the coverage vehicles. Since the method is focused on surface vehicles, navigation errors are not considered.

Spanning trees are a popular method for coverage planning [2], [3], [4]. These methods assume that either the navigation error is fixed or does not exist. This allows them to set an overlap parameter statically and plan the coverage motions accordingly. A growing error can be handled by setting the maximum error as the static error and creating the plan with the overlap it requires, this reduces the efficiency of the overall plan significantly.

In [5], the coverage path planning problem is considered with the goal of reducing total time to completion. As pointed out by the article, most vehicles are capable of higher speeds when traveling straight, thus minimizing the number of turns can lead to faster mission completion. Similarly, moving in straight lines can improve the navigation accuracy due to better studied dynamics, thus we also prefer linear motions in this paper.

In [6], a method is described such that an underwater vehicle balances between good navigational accuracy through re-visiting of landmarks and good coverage efficiency by reducing re-visitations. They handle these conflicting goals with a weighting parameter that is set by the user. In addition, they assume a non-flat salience map over the coverage area such that the vehicle can re-visit the highly interesting areas to limit its navigation error.
[7] examines a method in which the coverage area is divided into cells that can be efficiently covered by lawnmower patterns. The cell division is based on the known obstacles in the area that would otherwise disturb the lawnmower pattern. In [8], the coverage path planning problem is considered with the energy use of the vehicles in mind, taking into account the vehicle parameters. This allows for energy-efficient coverage paths to be generated. Most other works such as [9] also do not account for the uncertainty in robot pose estimations, and thus are a poor fit for underwater vehicles. For the interested reader, [10] provides a general survey into coverage path planning for robotics.

Navigation uncertainty must be accounted for when doing coverage. In [11], such an accounting method is described. The authors propagate the uncertainty in the vehicles pose estimate through its sensor model in order to compute a probabilistic coverage estimate. Coverage is defined in the form "with at least $\mathrm{X}$ probability, $\mathrm{Y}$ percent of the area is covered", where the parameters of area covered and with how much probability are mission parameters. The authors of [11] further show that this probabilistic model can be used to plan coverage paths that take into account the increasing inaccuracy of localization over long paths. They use this formulation to determine the distance between the lanes in a lawn-mower coverage pattern, assuming the navigation error will stay constant throughout the lane. In contrast, we do not have such an assumption and consider navigation inaccuracy to be growing continuously throughout the plan.

[12] compares the Extended Kalman Filter (EKFs)[13] against the Particle Filter (PF) [14] for IMU and sonar based sensor fusion for navigation. The result is that PFs are more accurate at the cost of heavy computational costs, thus both of them are still used. In the case of the EKF, the robot pose is a Gaussian random variable, whereas the PF keeps a population of hypotheses. In both cases, the robot pose diverges with distance traveled in a measurable way. Inspiration from such models can be used to estimate the upper bounds needed in this paper.

In [15], [16] methods of localization with acoustic range and bearing measurements are discussed. These works consider the localization problem by itself and do not incorporate a coverage mission. However, they are of interest in the case of multi-agent coverage planning where vehicles can act as landmarks for each other through acoustic measurements. Such a setup can be exceptionally beneficial if the seabed being covered has no features at all that can be used for navigation.

In this paper, we propose a method that takes into account the growth of the navigation uncertainty at the planning stage. We assume that the navigation method of choice used on the vehicle can be tested and its bounds can be approximated with a linear function of distance travelled. Note that such an error growth occurs e.g., when travelling with fixed speed through an underwater current of bounded magnitude. Using this assumption, our method then generates a coverage path plan that will guarantee complete coverage, even in the face of worst case navigation errors. 


\section{PROBLEM FORMULATION}

In this section we will define two problems that will be relating landmark separation and navigational error growth to coverage algorithms and efficiency. The difference between the problems is the assumptions regarding landmark separation, specified in Assumption 3 and 4. We start with an assumption of the worst case navigation error, and then define what we mean by the efficiency of a solution.

Assumption 1: The relative navigation error $e \in \mathbb{R}^{2}$ when traveling between two positions along a trajectory of length $d$ is bounded above as $\|e\| \leq k d$, where $k \in \mathbb{R}_{+}$.

Note that $k=0$ implies perfect navigation and $k=0.01$ implies a navigation error smaller than $1 \mathrm{~m}$ for every $100 \mathrm{~m}$ travelled.

Remark 1: Note that above, we assume upper bounds on the navigation error. Further note that we assume only translational errors, as rotational errors tend to have significantly tighter upper bounds on most vehicles equipped with a compass. An alternative formulation would be to assume that the navigation error was Gaussian. This is by far the most common assumption in robot navigation, and underlies many successful navigation concepts, from Kalman filters to graph SLAM. However, we believe that absolute bounds are reasonable for two reasons. First, one possible source for navigation errors in AUVs is underwater currents. These can be consistent over time and space, violating the Gaussian assumption of independently distributed errors. Second, we want to guarantee coverage, and since a Gaussian model includes non-zero (but small) probabilities for arbitrarily large errors, any analysis using a Gaussian model cannot guarantee coverage. Having noted this, a Gaussian study of this problem would be interesting, but is outside the scope of this paper.

Definition 1: If a planned trajectory of length $l$ guarantees coverage of an area $A$, using a sweep width of $w$, we define the efficiency of this trajectory as

$$
E=\frac{A}{l w} .
$$

Note that by the above definition, $E \in[0,1]$, and larger values are desirable, as they correspond to shorter paths that can be completed in less time. Also note that for AUVs, constant sweep width can be achieved by operation at a fixed height above the seafloor.

In order to guarantee coverage under navigation uncertainties, we first define our assumptions about the workspace. These definitions will later serve to show that if the vehicle can guarantee coverage of the smaller areas, then it can guarantee coverage of the larger area.

Assumption 2: There is a set of landmarks $L=\left\{L M_{i} \in\right.$ $\left.\mathbb{R}^{2}\right\}$, representing objects or locations that can be recognized. Together with Assumption 1 above, this implies that the relative navigation error between two AUV positions is bounded above as $\|e\| \leq k d_{L}$, where $d_{L}$ is the combined length of any two segments of the traversed trajectory that connect the two positions and the landmark.
Thus, covering a small area east of a landmark, visiting the landmark and then going for a long detour and coming back to the landmark before covering a small area north of the landmark gives the same relative navigation error between the two small areas as if the long detour did not happen. Note that a landmark could very well be another vehicle as long as it is stationary between visits. Such a setup would allow for the formulation to hold in places where there are no natural landmarks and potentially allow for a larger area to be covered.

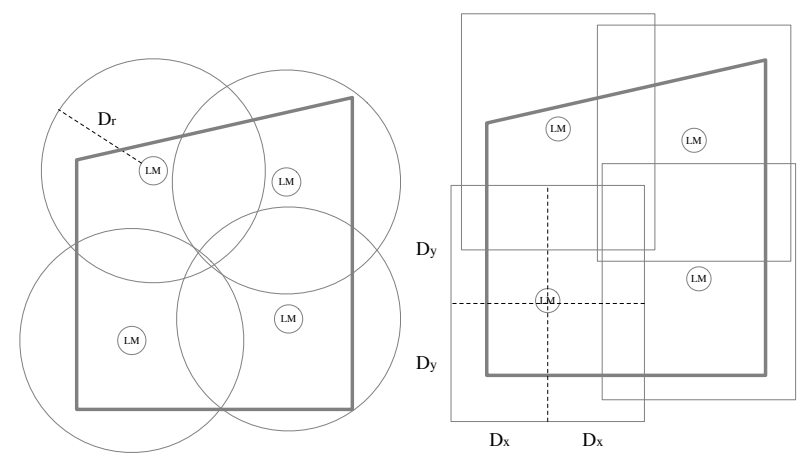

Fig. 2: A polygonal area $A$ of interest with four recognizable landmarks, $L M$. If $D_{r}$ is chosen as in the left figure, $A$ is completely covered by discs of radius $D_{r}$, centered at the landmarks. If $D_{x}, D_{y}$ are chosen as in the right figure, $A$ is completely covered by rectangles of size $2 D_{x}$ by $2 D_{y}$, centered at the landmarks.

Assumption 3: The area of interest $A$ can be covered by discs of radius $D_{r}$ centered at the landmarks $L$. That is, for every point $p \in A$, there is a landmark $L M \in L$ such that $\|p-L M\| \leq D_{r}$.

This assumption is illustrated in Figure 2.

Problem 1: If Assumptions 1, 2 and 3 above hold, for a given area $A$ of interest and disc radius $D_{r}$, what coverage algorithm can be applied to guarantee complete coverage, how large error growth $k$ can it handle and how efficient will it be?

Assumption 4: The area of interest $A$ can be covered by rectangles of width $D_{x}$ and length $D_{y}$ centered at the landmarks in $L$. That is, for every point $p \in A$ there exists a landmark $L M=\left(L M_{x}, L M_{y}\right) \in L$ such that $\left|p_{x}-L M_{x}\right| \leq D_{x}$ and $\left|p_{y}-L M_{y}\right| \leq D_{y}$.

This assumption is illustrated in Figure 2. Note that the orientation of the coordinate system is arbitrary, and can therefore be chosen with this assumption in mind when appropriate.

Problem 2: If Assumptions 1, 2 and 4 hold, for a given area $A$ of interest with maximal distances $D_{x}, D_{y}$ from any point in $A$ to a landmark in $L$. What coverage algorithm can be applied to guarantee complete coverage, and how large error growth $k$ can it handle and how efficient will it be?

The two problems defined above effectively divide the large coverage path planning problem into smaller chunks that can be solved individually. Thus we focus on solving 
the individual parts and computing their efficiencies, as they can then be combined to solve the full problem.

\section{PROPOSED APPROACH}

In this section we will describe three different algorithms for solving Problems 1 and 2 defined above.

\section{A. Covering using spiral patterns}

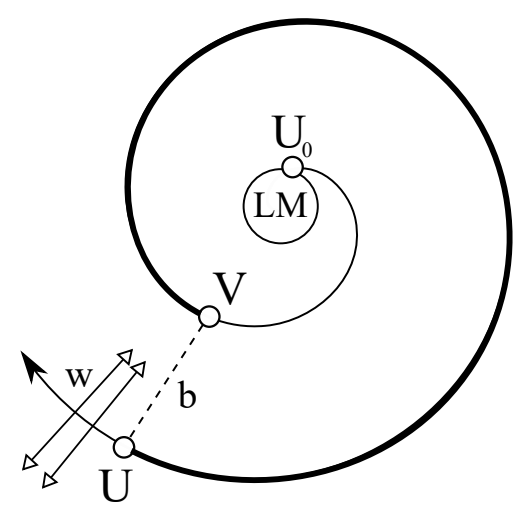

Fig. 3: A spiral sweep pattern with turn separation $b$ around a landmark $L M$. The sensor footprint $w$ is shown as doublearrows. $U$ and $V$ are two points on the spiral such that the angular difference between them is $2 \pi$. The round separation $b$ must account for the navigation error accumulated on the $\overparen{U V}$ arc. For points close to the landmark $L M$ such that there exists no point that comes $2 \pi$ before, $V=L M$.

Theorem 1 (Solving Problem 1): Given landmarks L, and error bounds $k$ according to Assumptions 1, 2 and 3. If the sensor footprint width is $w$, then area $A$ can be completely covered by applying a spiral pattern with radius $D_{r}$ centered at each landmark. The radius $D_{r}$ is dependent upon the sensor footprint $w$ and the error growth $k$. Let $V$ and $U$ be two arbitrary points on the spiral such that $U$ lies on a circle of radius $R_{U}$ and center $U_{0}$ while $V$ lies on the line segment between $U$ and $U_{0}$ (in angles, $U$ and $V$ are $2 \pi$ apart on the spiral). See Figure 3 . Let $\|U V\|$ be the arc length between points $U$ and $V$. Let $\theta$ be the progression angle on the spiral. The spiral with a rate of radius increase $\dot{R_{U}}=\dot{\theta}(w-\|\widehat{U V}\| k)$ covers a circle of radius $D_{r}$. Points that are on the "outside" of the spiral define the maximum range of guaranteed coverage. Let

$$
\mathcal{V}=\left\{V_{N 0}, V_{N 1}, \ldots\right\}
$$

be the set of such points on the spiral. The maximum radius of coverage is

$$
D_{r}=\min \left\{R_{V_{N}}+w / 2-\left\|U_{0} \overparen{V_{N}}\right\| k \mid V_{N} \in \mathcal{V}\right\} .
$$

The spiral pattern continues until $\|\overparen{U V}\| k=w$ where $R_{U}=$ $R_{V}$, the spiral self-intersects and does not generate more coverage from that point onward.

The efficiency of the spiral pattern that ends on point $U$ is simply

$$
E=\frac{A}{w l}=\frac{\pi D_{r}^{2}}{w\left\|\overparen{U_{0} U}\right\|}
$$

Due to the recursive nature of these formulations, a closed form solution is not available.

Proof: Let $R_{V}$ be the distance from $V$ to $U_{0}$. By definition, $R_{U}=R_{V}+b$ and $R_{U_{0}}=0$. In order to accommodate the error growth $k$ such that there will be no gaps between $U$ and $V$, the relative error accumulated over the distance traveled $\|\overparen{U V}\|$ must be equal to the sensor width $w$, thus the spiral separation distance $b$ must be

$$
0 \leq b \leq w-\|\widehat{U V}\| k
$$

$R_{U} \geq R_{V}+w-\|\overparen{U V}\| k$ follows from above. This guarantees that the inside of the spiral is covered regardless of the outer radius.

In order to determine the maximum coverage radius $D_{r}$, we consider the two types of points on the spiral; Points that are in pairs of $(U, V)$ such that the point $V$ has a paired point $U$ that is $2 \pi$ after it, and points that do not have such a pairing. Points that are paired, guarantee gapless coverage inside the spiral according to Eq. 5. The maximum coverage radius is thus defined by these unpaired points on the "outside" of the spiral.

For any point $V_{N} \in \mathcal{V}$ (As defined in Eq. 2), the total accumulated error is $\epsilon_{N}=\left\|U_{0} \overparen{V_{N}}\right\| k$, which in the worst case is oriented in the direction of $U_{0}$ from $V_{N}$. In this worst case, the outward reach of $V_{N}$ is $R_{V_{N}}+w / 2-\epsilon_{N}$. Thus, the maximum radius of coverage is $D_{r}=\min \left\{R_{V_{N}}+w / 2-\right.$ $\left.\left\|U_{0} \overparen{V_{N}}\right\| k \mid V_{N} \in \mathcal{V}\right\}$.

For the last point $U$ on the spiral, the total distance of the spiral is $l=\left\|\overparen{U_{0} U}\right\|$, thus the work done by the AUV is $w\left\|\overparen{U_{0} U}\right\|$. Equation 4 follows from Eq. 1.

\section{B. Covering using circular patterns}

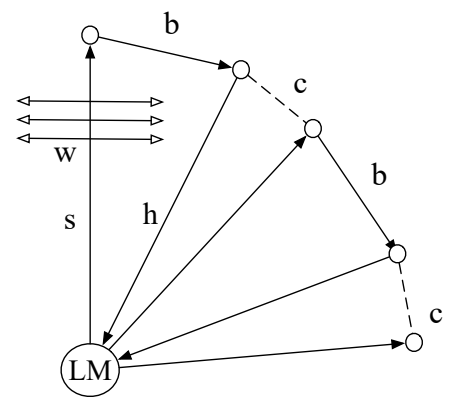

Fig. 4: A circular sweep pattern of radius $s$ around a landmark $L M$. The sensor footprint width is $w$ and the sweep separations at the edge of the pattern are $b$ when switching from outward to inward sweeps and $c$ when switching from inward to outward. Note that $c$ is significantly smaller than $w$ as it has to account for the absolute navigation error accumulated when going back to the center and out to the edge again while $b$ only needs to account for the relative error. The localization uncertainty is reset every time the vehicle sees the landmark.

Theorem 2 (Solving Problem 1): Given landmarks $L$, and error bounds $k$ according to Assumptions 1, 2 and 3. If the 
sensor footprint width is $w$, then area $A$ can be completely covered by applying a pattern of radius $s$ centered at each landmark. While the inward and outward sweeps $s$ and $h$ can be set differently in general, we set $s=h$ to keep the circular shape. The sweep pattern is illustrated in Figure 4, with angular progression determined by the sweep separation distances $b, c$ as

$$
\begin{aligned}
& s=D_{r} /(1-k), \\
& b=w /(1+k), \\
& c=w-(2 s+b) k .
\end{aligned}
$$

The pattern will furthermore lead to the efficiency

$$
E=\frac{\pi^{2} D_{r}^{3}\left(2 D_{r} k+2 D_{r}-k w+w\right)}{w\left(D_{r} k^{3}-D_{r} k+k^{2} w-2 k w+w\right)}
$$

for each disc, and an upper bound on pattern radius as a function of the error growth $k$ and sweep width $w$ is given by

$$
D_{r} \leq \frac{w(k-1)^{2}}{4 k(k+1)}
$$

Proof: First we note that aiming for a radius of $s$, the navigation error at the edge of the disk is at most $s k$ giving a guaranteed radius of $D_{r}=s-s k=s(1-k)$. Second, to avoid a gap between the sweeps before and after $b$ we need the error bound $k b$ to equal the overlap of the sensor sweeps, that is $k b=w-b$, which gives $b=w /(1+k)$.

Third, to avoid a gap between the sweeps before and after visiting the landmark, the overlap $w-c$ needs to be equal to the error bound $(2 s+b) k$, that is $w-c=(2 s+b) k$, which gives $c=w-(2 s+b) k$.

Finally, to complete the lap around the landmark and cover the whole disc we need $N$ excursions from the landmark, each progressing $b+c$ along the disc boundary of length $2 s \pi$ that is $N(b+c)=2 s \pi$, which gives

$$
N=\frac{\pi D_{r}(1+k)}{w-D_{r} k^{2}-D_{r} k-k w}
$$

The efficiency is thus

$$
\begin{aligned}
E & =\frac{A}{w l}=\frac{D_{r}^{2} \pi}{w N(2 s+b)} \\
& =\frac{\pi^{2} D_{r}^{3}\left(2 D_{r} k+2 D_{r}-k w+w\right)}{w\left(D_{r} k^{3}-D_{r} k+k^{2} w-2 k w+w\right)}
\end{aligned}
$$

Given the vehicle must be guaranteed to detect the landmark after each excursion of length $(2 s+b)$, the navigation error $(2 s+b) k$ must be less than half of the sensor width, $(2 s+b) k \leq w / 2$. Setting $s=D_{r} /(1-k)$ and $b=w /(1+k)$ and solving for $D_{r}$ yields

$$
D_{r} \leq \frac{w(k-1)^{2}}{4 k(k+1)}
$$

Note that with no navigation errors, $k=0$ would imply that no overlap in the sweeps would be required, i.e., $b=c=$ $w$, and arbitrarily large discs can be covered as $D_{r \max } \rightarrow \infty$.
But with $k>0$ we must choose $b, c<w$ to have an overlap that accounts for the navigation errors. This in turn limits the disc radius where the progression gained by a sweep is equal to the overlap needed to account for the worst case navigation error.

Finally, note that the circular pattern in Figure 4 has inherent overlaps near the landmark. Thus, even at ideal navigation with $k=0$, and minimal sensor width $w \rightarrow 0$ efficiency is $E_{\max } \rightarrow 1 / 2$. For example, when $D_{r}=100$, $w=1$ and $k=0, E=100 / 201$. This leads us to investigating the efficiency of rectangular patterns.

\section{Covering using Rectangular Patterns}

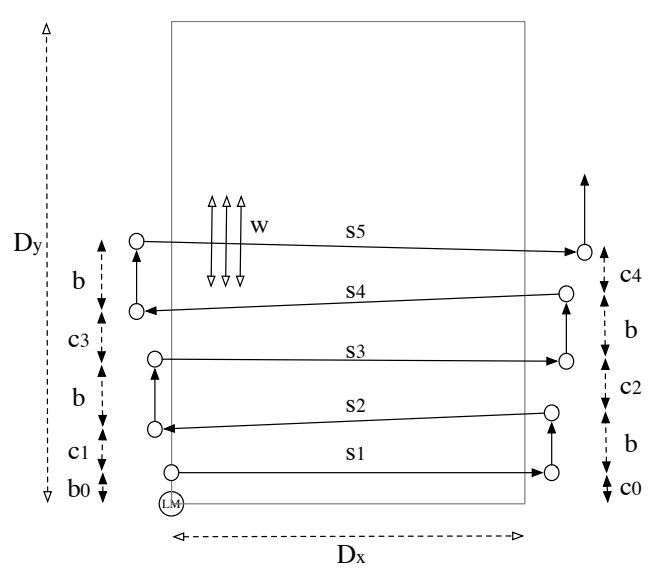

Fig. 5: The sweep pattern for covering a patch of size $D_{x} \times D_{y}$. Note that this pattern does not return to the landmark (LM) to reset the navigation error, thus the error keeps growing, resulting in increasing sweep widths $s_{i}$ to guarantee coverage of the patch. Furthermore, when $s_{i}$ grows, one of the sweep separation parameters $c_{i}$ shrinks to guarantee overlap, while the other, $b$, is constant.

Theorem 3 (Solving Problem 2 (part 1)): Given landmarks $L$, and error bounds $k$ according to Assumptions 1,2 and 4. If the sensor footprint width is $w$, then the area $A$ can be completely covered by creating four rectangular patches of size $D_{x} \times D_{y}$ around each landmark. Each of these patches are furthermore covered by a lawn mower sweep pattern, as illustrated in Figure 5, with increasing sweep lengths $s_{i}$ and sweep separation distances $b, c_{i}$ given below

$$
\begin{aligned}
b_{0} & =\frac{w}{2}, \\
s_{1} & =\frac{D_{x}}{1-k}, \\
c_{0} & =\frac{w}{2}-s_{1} k \\
b & =w /(1+k), \\
s_{i+1} & =\frac{1+k}{1-k} s_{i}+\frac{k}{1-k} b, \\
c_{i} & =w-k\left(s_{i+1}+s_{i}+b\right) .
\end{aligned}
$$



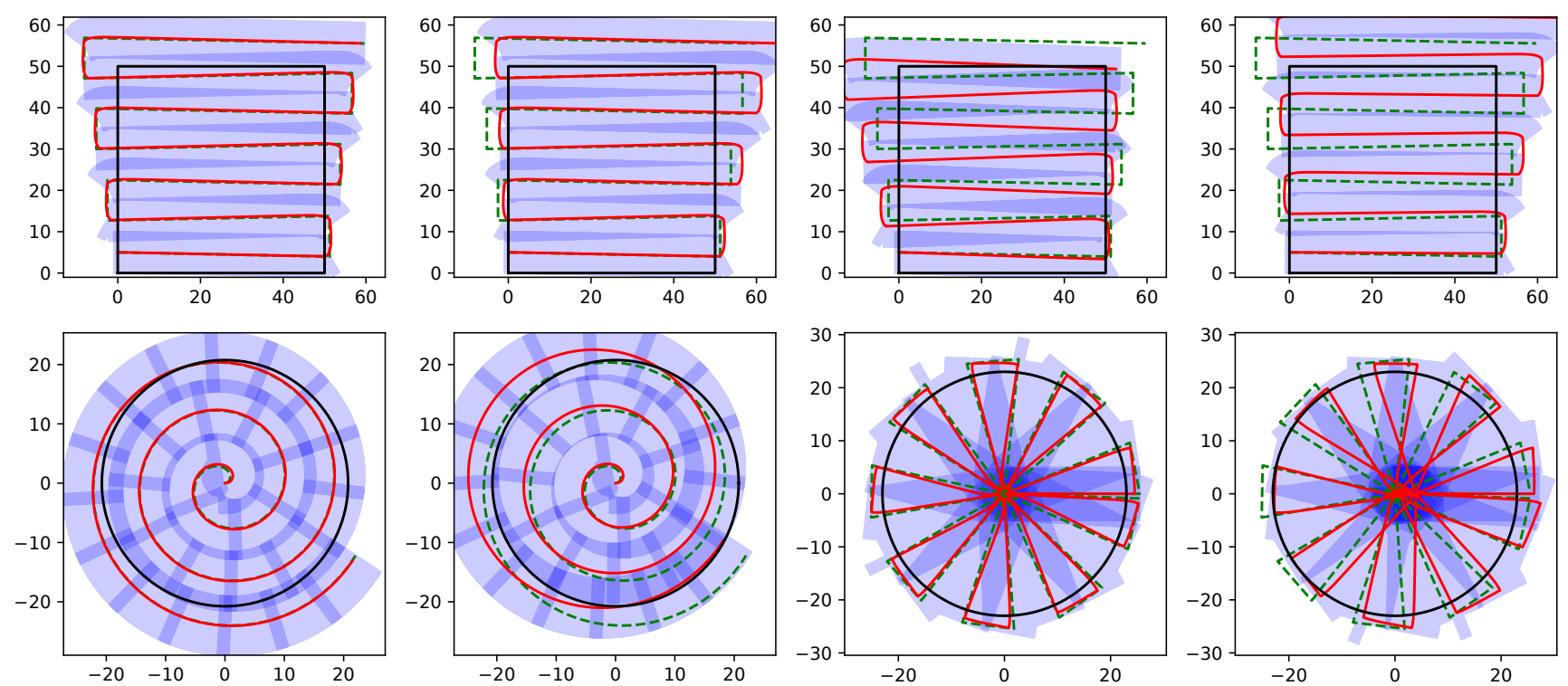

Fig. 6: Simulations of the three different coverage patterns. First row, left to right: Rectangular pattern with no drift, drift only in $+x$ direction, drift in $-x,-y$ direction, and drift in $+x,+y$ directions. Second row: Spiral and Circular patterns. Left to right: Spiral with no drift, spiral with drift towards $-x,+y$, Circular with no drift, Circular with drift towards $+x$. All values in meters. Top-down perspective. Green dashed line shows the planned pattern, red solid line shows the trajectory of the AUV and the solid black rectangle or circle shows the guaranteed coverage area. Blue shaded areas show the covered areas, with darker shades indicating overlap. The Spiral pattern also shows equidistant parts with perpendicular-to-motion dark-shaded areas. $w=20$ and $k=0.02$ for all experiments.

The number of sweeps $N$ needed to cover the patch $D_{x} \times D_{y}$ can be found by solving

$$
D_{y}=\Sigma_{i=1}^{N / 2} c_{2 i-1}+b-\left(s_{2 i-1}+s_{2 i}+2 b\right) k .
$$

The corresponding efficiency is

$$
E=\frac{D_{x} D_{y}}{\sum_{i=1}^{N / 2}\left(s_{2 i-1}+s_{2 i}+2 b\right) w}
$$

Note that $s_{i}$ increases exponentially. Thus, it is clear that for each area width $D_{x}$, the efficiency will decrease with increasing patch height $D_{y}$, and there is an upper bound on the patch height $D_{y}$ that can be covered. It is straightforward to compute this upper bound from (14)-(21) above, but due to the recursive computations involved, there is no closed form expression for it.

Proof: First, to avoid a gap between the sweeps before and after $b$ we need the error bound $k b$ to equal the overlap of the sensor sweeps, that is $k b=w-b$, which gives $b=$ $w /(1+k)$.

Second, to avoid a gap between the sweeps on different sides of $c_{i}$, the overlap $w-c_{i}$ needs to be equal to the error bound $k\left(s_{i+1}+s_{i}+b\right)$, that is $w-c_{i}=k\left(s_{i+1}+s_{i}+b\right)$, which gives $c_{i}=w-k\left(s_{i+1}+s_{i}+b\right)$.

To provide overlap on the side in the first sweep we have $s_{1}=D_{x}+s_{1} k$ which gives $s_{1}=D_{x} /(1-k)$. In the following sweeps we have that the next sweep should equal the previous one, plus a margin for the relative error, thus $s_{i+1}=s_{i}+k\left(s_{i+1}+s_{i}+b\right)$ which gives (18).
The number of sweeps needed is given by just summing the progress along the left hand side of Figure 5.

\section{Illustration of results}

To illustrate that the proposed coverage pattern does indeed cover the desired area, even in the presence of worst case constant error drift in one direction, we simulate trajectories in Figure 6. In all three patterns, the gap between the planned coverage path and the actual path constantly increases due to the drift, however, the area is still covered completely.

The efficiencies of the three approaches are illustrated in Figures 7, 8, 9 and 10. In Figure 7, the efficiency of the spiral pattern with respect to the maximum coverage radius is shown. As expected, the efficiency is low in the beginning, due to large amount of overlap at the center of a very small spiral $\left(D_{r}<w\right)$. However, the spiral can reach quite high efficiency values under very specific circumstances. Unfortunately, the efficiency starts dropping rapidly right after its peak, resulting in a narrow range of operation parameters.

As can be seen in Figure 8, the efficiency of the circular pattern approaches its theoretical maximum of $1 / 2$, due to large overlaps near the landmark, at low $k$ values. However, the efficiency of the circular pattern falls very slowly with increasing $D_{r}$ values. Thus, compared to the spiral pattern, the operation limits of the circular pattern are significantly 


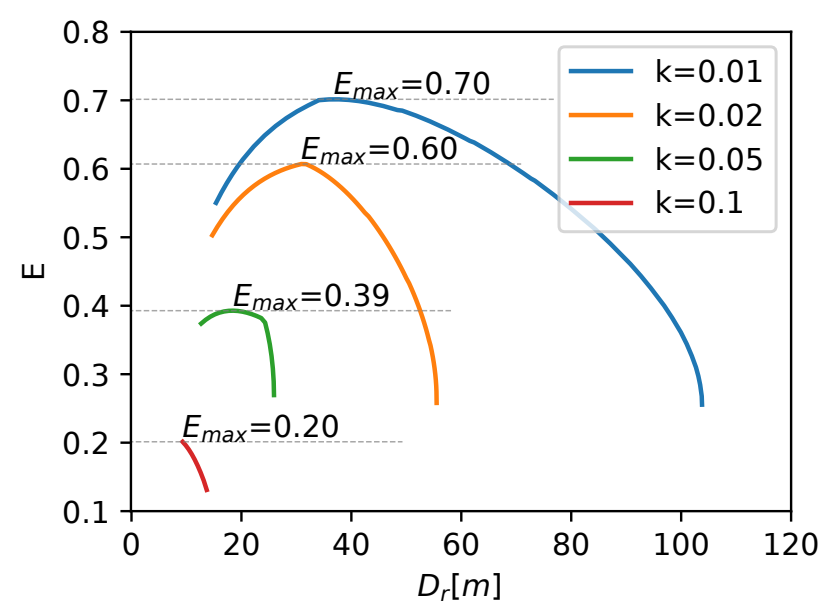

Fig. 7: Efficiency of the spiral pattern. While the efficiency of this pattern can reach relatively high values, its effective maximum radius is significantly lower compared to the circular pattern. The sweep width is set to $w=20$.

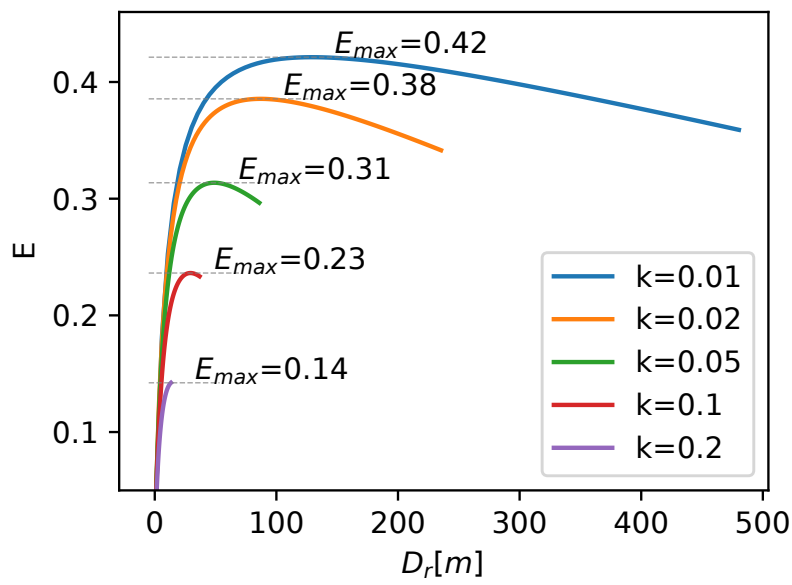

Fig. 8: Efficiency of circular pattern starts low due to the overlap in the center, but decreases slowly with radius, as the navigation error is reset every time the AUV passes the landmark in the middle. The sweep width is set to $w=20$.

larger. The different upper bounds of $D_{r}$ can also be observed for different $k$ values. As expected, lower error growth rates allow for more efficient coverage and larger areas to be covered.

In Figure 9, the efficiency of the rectangular pattern with respect to the sides of the rectangle is shown. As can be seen in the figure, the efficiency shows a stepping behavior. This behavior is expected, since when the rectangular pattern adds a new row to the path, the efficiency is drastically lowered, followed by a linear climb until a new row is needed again. The harshness of this stepping behavior also changes with $D_{x}$. The wider the pattern is, the more frequent the jumps become with increased $D_{y}$. This effect is explained by the larger accumulated navigation error on individual rows with large $D_{x}$ values.

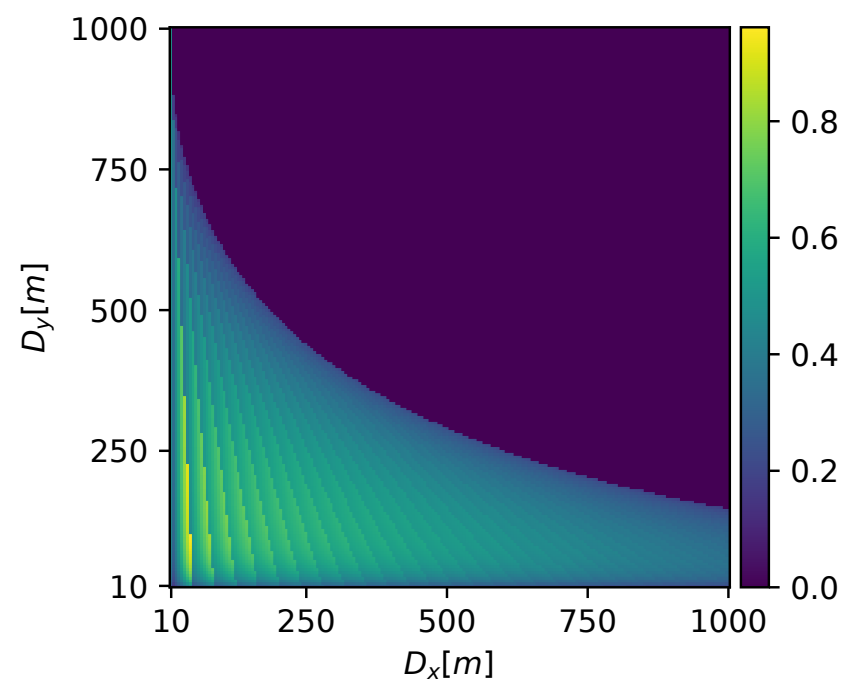

Fig. 9: Efficiency of the rectangular pattern(the brighter the color, the more efficient) is high for small areas due to small overlap, but decreases rapidly with width and height of the area as increasing error bound leads to larger margins. The stepping behavior of the efficiency is clearly visible. The sweep width and error growth rate are set to $w=20$ and $k=0.01$ respectively.

In Figure 10, the efficiency of the three patterns are compared with respect to equal amounts of coverage. The rectangular pattern is set to be a square of sides $D_{r}$. As can be seen in the figure, the rectangular pattern reaches higher maximum efficiency values compared to the circular pattern at the cost of total area covered. Comparatively, the spiral pattern can achieve efficiencies in between the circular and rectangular patterns in a limited operational area in a more stable manner than the rectangular pattern. The spiking nature of the rectangular pattern can also be observed in this figure. On the other hand, the circular coverage pattern can cover significantly larger areas, at the cost of doing so at half the efficiency. This extra coverage capability is due to the navigation error resets at the central landmark which also introduces sensor overlaps that reduce the efficiency.

\section{CONCLUSIONS}

In this paper, we have analysed the three most common coverage patterns, the rectangular (lawnmower) pattern, the spiral and the circular (radial) pattern. We have shown how to adapt the three patterns to a given navigation-error growthrate in order to guarantee complete coverage. We have also computed how the efficiency decreases as a function of pattern size and error-growth and finally showed how this efficiency can be translated into bounds on the landmark sparsity in the regions to be covered.

Our analysis has shown that using the circular pattern is best when the area to be covered is very large, at the cost of efficiency. This way of coverage is better suited for vehicles with long range and long endurance properties, such as LoLo[17](Fig. 11) and Autosub [18]. Whereas if the area 


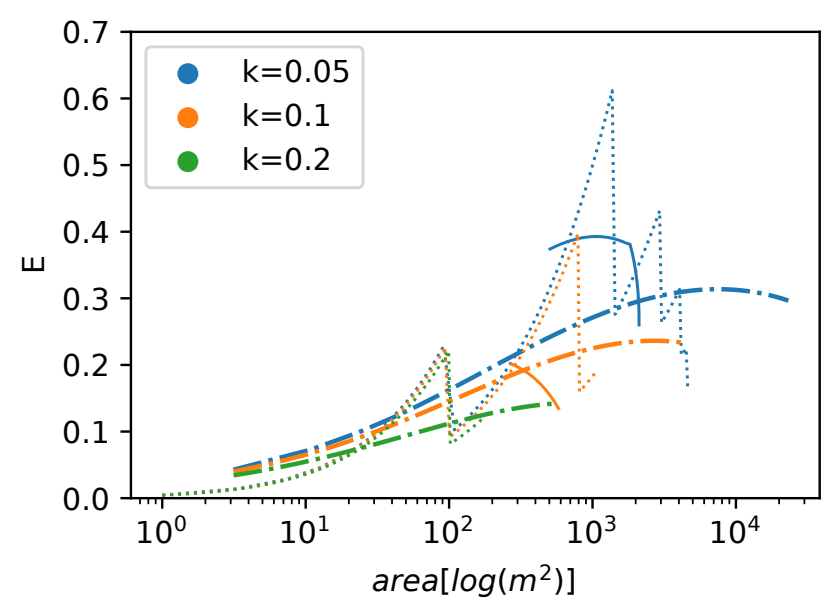

Fig. 10: Comparing efficiencies between a disc and a square shaped area, using the three patterns. Dotted lines show the rectangular pattern, dash-dotted lines show the circular pattern and the solid lines show the spiral pattern. Colors indicate uncertainty growth rates for $k=0.05, k=0.1$ and $k=0.2$. Sensor width $w=20$ for all.

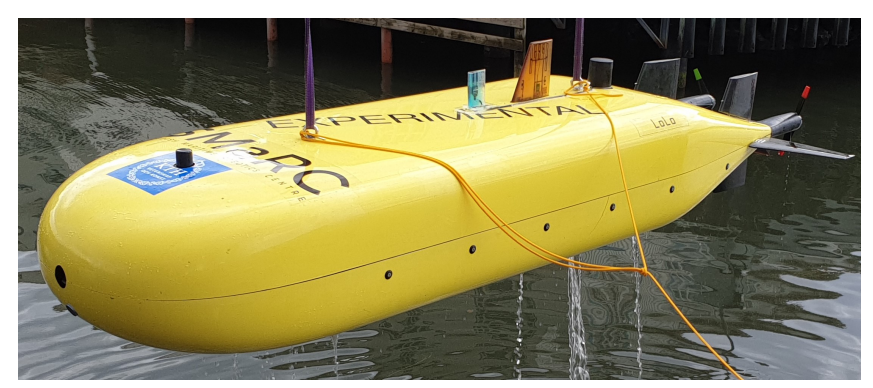

Fig. 11: The LoLo AUV being developed as part of the SMaRC Project.

to be covered is relatively smaller, it can be covered almost twice as efficiently with the rectangular pattern instead. This mode of operation is better suited for multiple smaller vehicles such as [19]. These two scenarios correspond to areas with more and fewer landmarks. In most situations, the spiral pattern was outperformed by the other two methods, furthermore it had the most limited operational bounds and thus is not recommended for general use.

As future work, we will test our findings experimentally within the SMaRC Project ${ }^{1}$, and compare our results to the theoretical and simulated results shown in this paper. The experimental platform LoLo (Fig. 11)is foreseen as the vehicle to be used and is nearing completion.

\section{ACKNOWLEDGMENT}

This work was supported by Stiftelsen for Strategisk Forskning (SSF) through the Swedish Maritime Robotics Center (SMaRC) (IRC15-0046).

\section{REFERENCES}

[1] Nare Karapetyan, Jason Moulton, Jeremy S Lewis, Alberto Quattrini $\mathrm{Li}$, Jason M O'Kane, and Ioannis Rekleitis. Multi-robot dubins coverage with autonomous surface vehicles. In 2018 IEEE International Conference on Robotics and Automation (ICRA), pages 2373-2379. IEEE, 2018.

[2] Yoav Gabriely and Elon Rimon. Spanning-tree based coverage of continuous areas by a mobile robot. Annals of mathematics and artificial intelligence, 31(1):77-98, 2001.

[3] Guan-Qiang Gao and Bin Xin. A-stc: auction-based spanning tree coverage algorithm formotion planning of cooperative robots. Frontiers of Information Technology \& Electronic Engineering, 20(1):18-31, 2019.

[4] Chunqing Gao, Yingxin Kou, Zhanwu Li, An Xu, You Li, and Yizhe Chang. Optimal multirobot coverage path planning: ideal-shaped spanning tree. Mathematical Problems in Engineering, 2018, 2018.

[5] Isaac Vandermeulen, Roderich Groß, and Andreas Kolling. Turnminimizing multirobot coverage. In 2019 International Conference on Robotics and Automation (ICRA), pages 1014-1020. IEEE, 2019.

[6] Ayoung Kim and Ryan M Eustice. Perception-driven navigation: Active visual slam for robotic area coverage. In 2013 IEEE International Conference on Robotics and Automation, pages 3196-3203. IEEE, 2013.

[7] Anqi Xu, Chatavut Viriyasuthee, and Ioannis Rekleitis. Efficient complete coverage of a known arbitrary environment with applications to aerial operations. Autonomous Robots, 36(4):365-381, 2014.

[8] Carmelo Di Franco and Giorgio Buttazzo. Energy-aware coverage path planning of uavs. In 2015 IEEE international conference on autonomous robot systems and competitions, pages 111-117. IEEE, 2015.

[9] Ioannis Rekleitis, Vincent Lee-Shue, Ai Peng New, and Howie Choset. Limited communication, multi-robot team based coverage. In IEEE International Conference on Robotics and Automation, 2004. Proceedings. ICRA'04. 2004, volume 4, pages 3462-3468. IEEE, 2004.

[10] Enric Galceran and Marc Carreras. A survey on coverage path planning for robotics. Robotics and Autonomous systems, 61(12):12581276, 2013.

[11] Liam Paull, Mae Seto, and Howard Li. Area coverage planning that accounts for pose uncertainty with an auv seabed surveying application. In 2014 IEEE International Conference on Robotics and Automation (ICRA), pages 6592-6599. IEEE, 2014.

[12] G Rigatos and G Gerasimos. Extended kalman and particle filtering for sensor fusion in motion control of mobile robots. Mathematics and computers in simulation, 81(3):590-607, 2010.

[13] G Rigatos and S Tzafestas. Extended kalman filtering for fuzzy modelling and multi-sensor fusion. Mathematical and computer modelling of dynamical systems, 13(3):251-266, 2007.

[14] M Sanjeev Arulampalam, Simon Maskell, Neil Gordon, and Tim Clapp. A tutorial on particle filters for online nonlinear/non-gaussian bayesian tracking. IEEE Transactions on signal processing, 50(2):174$188,2002$.

[15] Alexander Bahr, Matthew R Walter, and John J Leonard. Consistent cooperative localization. In 2009 IEEE International Conference on Robotics and Automation, pages 3415-3422. IEEE, 2009.

[16] Alexander Bahr, John J Leonard, and Maurice F Fallon. Cooperative localization for autonomous underwater vehicles. The International Journal of Robotics Research, 28(6):714-728, 2009.

[17] Clemens Deutsch, Lázaro Moratelli, Sebastian Thuné, Jakob Kuttenkeuler, and Filip Söderling. Design of an auv research platform for demonstration of novel technologies. In 2018 IEEE/OES Autonomous Underwater Vehicle Workshop (AUV), pages 1-8. IEEE, 2018.

[18] Daniel T Roper, Alexander B Phillips, Catherine A Harris, Georgios Salavasidis, Miles Pebody, Robert Templeton, Sriram Vikraman Sithalashmi Amma, Micheal Smart, and Stephen McPhail. Autosub long range 1500: An ultra-endurance auv with $6000 \mathrm{~km}$ range. In OCEANS 2017-Aberdeen, pages 1-5. IEEE, 2017.

[19] Sriharsha Bhat, Ignacio Torroba, Özer Özkahraman, Nils Bore, Christopher Iliffe Sprague, Yiping Xie, Ivan Stenius, Josefine Severholt, Carl Ljung, John Folkesson, et al. A cyber-physical system for hydrobatic auvs: system integration and field demonstration. In 2020 IEEE/OES Autonomous Underwater Vehicles Symposium $(A U V)(50043)$, pages 1-8. IEEE, 2020. 